\title{
THE EFFECT OF CO-EXISTENT STIMULI OTHER THAN TEST STIMULUS ON BRIGHTNESS CONSTANCY ${ }^{1}$
}

\author{
AIKO KOZAKI \\ Tokyo Woman's Christion College
}

\begin{abstract}
The purpose of this study is to investigate the relationship between constancy and contrast concerning such factors as the co-existence of the second inducing field (Exp. I), the structure of the visual field (Exp. II), and the existence of the additional stimuli other than the test and inducing fields (Exp. III). As the stimuli pieces of achromatic paper were used. In Experiment I a binocular matching technique by means of the method of limits was adopted. In Experiments II and III brightness judgments were done by absolute term using 9 adjective categories. The results supported the view that constancy does not vary as a linear function of contrast. Discussion was made on the role of lighter stimuli in constancy.
\end{abstract}

Historically (Gelb, 1930; Kardos, 1934; Koffka, 1932; Wallach, 1948), brightness constancy has been considered to depend upon the co-existence of stimuli other than the test stimulus in the visual field. Moreover, the recent studies of brightness constancy generally agree with respect to that the background brightness is the most important factor concerning brightness constancy, i.e., that the degree of the constancy depends upon the reflectance of the background or the inducing field (Hano, 1959; Helson, 1938 and 1943; Kozaki, 1963; Leibowitz, 1955). Such results lead to the assumption that the simultaneous brightness contrast plays a major role in brightness constancy. Especially, on the basis of experimental findings Leibowitz (1955, and 1957) and Dunn \& Leibowitz (1961) assumed that there was co-variant relationship between brightness con-

1 This article is based on parts of a dissertation submitted in partial fulfillment of the requirements for the Ph. D. Dergree in Department of Psychology, Keio University. The writer is indebted to Prof. T. Ogawa of Keio University for his advices offered throughout the course of the experimental investigation. Special thanks are also offered to Dr. Y. Takada of Tokyo Woman's Christian College for a critical reading of the manuscript. stancy and simultaneous contrast. Our recent experiments (Kozaki, 1963) suggested, however, that the relationship between brightness constancy and contrast was nonlinear, the degree of constancy being unproportionately affected by the degree of contrast. In this study the relationship between brightness constancy and contrast was investigated by simultaneous measurement of both phenomena with respect to the common variables such as the reflectance of the inducing field and the area of the test field and of the inducing field. And the results showed three significant facts about the relationship between brightness constancy and contrast. First, there was greater constancy when the reflectance of the inducing field exceeded that of the test field than when inducing reflectance was lower than test field reflectance; this result was accordant with Leibowitz's findings. Secondly, though contrast was varied by increasing the reflectance of the inducing field, constancy was invariant. Third, as to the area of the test field and of the inducing field, the change of these areas produced no definite effects on the degree of constancy, while it affected the degree of contrast. These second and third points were important aspects of the results. 
It is certain that constancy is influenced by the co-existent stimuli in the visual field and in especially promoted by those which have higher reflectance than the test stimulus. However, our experiments indicated that contrast was not always associated with constancy. In the previous paper (Kozaki, 1963) it was pointed out that more detailed studies of the relationship between brightness constancy and contrast were necessary. The present study is planned to explore further the relationship between constancy and contrast by examining such variables as the coexistence of the second inducing field, the test field areas covering the wider range, the stimulus field condition (the structure of the visual field), and the insertion of the cue stimulus into the visual field.

\section{EXPERIMENT I}

\section{Purpose}

The purpose of the experiment is to examine the effect of the second inducing field by which the test and the first inducing fields are surrounded on the apparent brightness of the test field under various levels of illuminance.

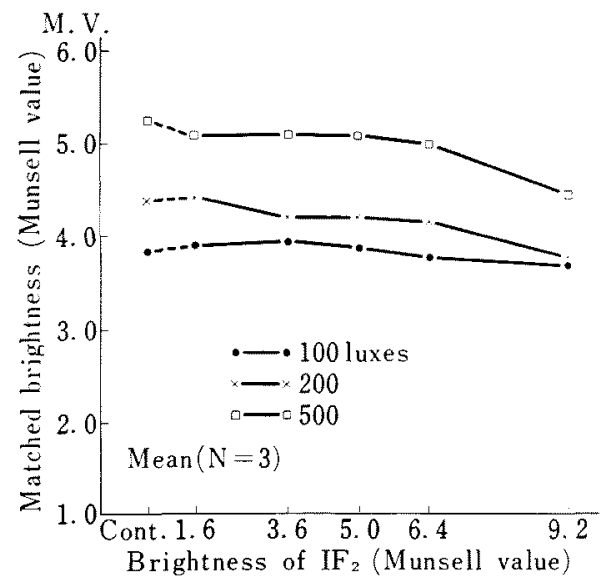

FIG. 1. Matched brightness as a function of the brightness of the second inducing field $\left(\mathrm{IF}_{2}\right)$ is plotted with the illuminance as the parameter (in the case of $\mathrm{IF}_{2}$ : $\left.1.6 \mathrm{M} . \mathrm{V}.\right)$. Each datum is the mean of 3 Os.

\section{Apparatus and Procedure}

The apparatus was similar to that used in the previous study (Kozaki, 1963). The $O$ was in the dark box attached to the two viewing windows in the observation tube. The $O$ when placing his chin on the chin rest could monocularly see the standard and comparison fields through each window. The standard field consisted of a square test field and two inducing fields that surrounded the test field concentrically. The test field subtended a visual angle of $1^{\circ} 3^{\prime}$ and each width of inducing field subtended $36^{\prime}$ and $1^{\circ} 3^{\prime}$ respectively at a viewing distance of $140 \mathrm{~cm}$. The test field of the standard was kept at constant neutral gray (Munsell Value $5: 19.77 \%$ reflectance). The inner inducing field $\left(\mathrm{IF}_{1}\right)$ had either one of two levels of reflectance $: 2.21 \%(1.6 \mathrm{~V})$ or $34.92 \%$ $(6.4 \mathrm{~V})$, whereas the outer inducing field $\left(\mathrm{IF}_{2}\right)$ had the following five levels of reflectance: $2.21 \%(1.6 \mathrm{~V}), 9.55 \%(3.6 \mathrm{~V}), 19.77 \%(5.0 \mathrm{~V})$, $34.92 \%(6.4 \mathrm{~V})$ or $83.07 \%(9.2 \mathrm{~V})$. The comparison fields consisted of the matching field of the same size as the test field and the background which subtended a visual angle of $5^{\circ} 18^{\prime}$ $\times 5^{\circ} 18^{\prime}$. While the background was kept at the constant reflectance level $(3.2 \%)$, the matching field was varied in terms of 0.2 Munsell Value steps by a series of gray papers. Measurements were undertaken by the method of limits. After the 20 min dark adaptation the $O$ s

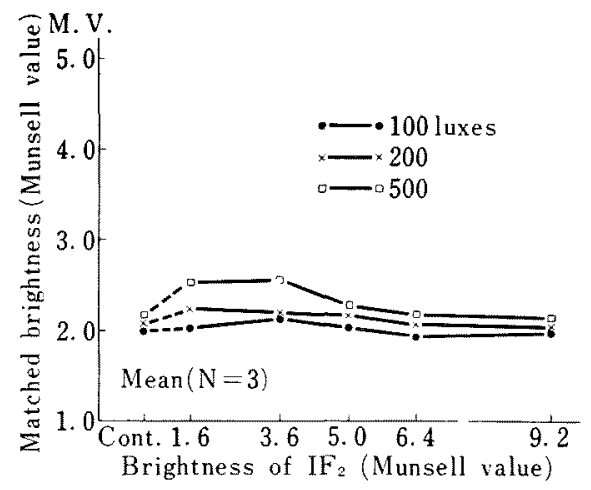

FIG, 2. Matched brightness as a function of the brightness of the second inducing field $\left(\mathrm{IF}_{2}\right)$ is plotted with the illuminance as the parameter (in the case of $\left.\mathrm{IF}_{1}: 6.4 \mathrm{M} . \mathrm{V}.\right)$. Each datum in the mean of $3 \mathrm{Os}$. 
Analysis of variance for the data in Figs. 1 and 2

Table $1 \mathrm{a}, \mathrm{IF}_{1}$ : 1.6 Munsell Value

\begin{tabular}{|c|c|c|c|c|}
\hline $\begin{array}{l}\text { Source of } \\
\text { variation }\end{array}$ & $S S$ & $d f$ & $M S$ & $F$ \\
\hline $\begin{array}{l}\text { Lightness of } \\
\mathrm{IF}_{2} \text { (L) }\end{array}$ & 0.4986 & 4 & 0.1247 & 2.0309 \\
\hline Subjects (S) & 2.4622 & 2 & 1.2311 & $2.0050 * *$ \\
\hline $\begin{array}{l}\text { Difference of } \\
\text { Illuminance (I) }\end{array}$ & 4.1739 & 1 & 4.1739 & $67.9780^{* *}$ \\
\hline$L \times S$ & 0.7083 & 8 & 0.0885 & $1.44 \mathrm{I}$ \\
\hline $\mathbf{L} \times \mathbf{I}$ & 0.1887 & 4 & 0.0472 & \\
\hline $\mathrm{S} \times \mathbf{I}$ & 0.9913 & 2 & 0.4957 & $8.0717^{*}$ \\
\hline $\mathrm{L} \times \mathrm{S} \times \mathrm{I}$ & 0.4912 & 8 & 0.0614 & \\
\hline Total & 9.5142 & 29 & & \\
\hline
\end{tabular}

matched the matching field, which was at the constant level of illuminance throughout the experiment (200 luxes), with the test field at the following illuminance levels : 500, 200 and 100 luxes. The standard and comparison fields were observed successively by means of the binocular matching method. Data were obtanned from three $O$ s who are undergraduate students of psychology at Tokyo Woman's Christian College.

\section{Results}

The matched brightness of the comparison stimuli under six conditions of the outer inducing field reflectance and under three levels of illuminance are shown in Figs. 1 and 2. Fig. 1 shows the result when the reflectance of the inner inducing field is $2.21 \%(1.6 \mathrm{~V})$, while Fig. 2, when it is $34.92 \%(6.4 \mathrm{~V})$. In these figures perfect constancy will be shown by the coincidence of three curves corresponding to the three illuminance levels of 500, 200 and 100 luxes. Results reveal two important facts. In the first place, irrespective of the reflectance of $\mathrm{IF}_{2}$ the tendency of coincidence of three curves is more prominent in Fig. 2 than in Fig. 1 ; i.e., the tendency towards constancy is greater in the former than in the latter. This result is consistent with the past findings about the relationship between constancy of the test field and the reflectance of the inducing field. Sec-
Table $\mathrm{lb}, \mathrm{IF}_{1}: 6.4$ Munsell Value

\begin{tabular}{ccrcc}
\hline $\begin{array}{c}\text { Source of } \\
\text { variation }\end{array}$ & $S S$ & $d f$ & $M S$ & $F$ \\
\hline $\mathrm{L}$ & 0.2798 & 4 & 0.0699 & $3.9452^{*}$ \\
$\mathrm{~S}$ & 0.0728 & 2 & 0.0364 & 2.0530 \\
$\mathrm{I}$ & 0.2651 & $\mathbf{1}$ & 0.2651 & $14.952^{* *}$ \\
$\mathrm{~L} \times \mathrm{S}$ & 0.4536 & 8 & 0.0567 & 3.1979 \\
$\mathrm{~L} \times \mathrm{I}$ & 0.0466 & 4 & 0.0117 & \\
$\mathrm{~S} \times \mathrm{I}$ & 0.0765 & 2 & 0.0383 & 2.1573 \\
$\mathrm{~L} \times \mathrm{S} \times \mathrm{I}$ & 0.1419 & 8 & 0.0177 & \\
Total & 1.3363 & 29 & &
\end{tabular}

ondly, inspection of the curves in Figs. 1 and 2 suggests that there is some effect of the outer inducing field on the apparent brightness of the test field, even though the amount of the effect is small. In order to examine the effect of $\mathrm{IF}_{2}$ on constancy analyses were performed on the data of Conditions $\mathrm{A}\left(\mathrm{IF}_{1}: 1.6 \mathrm{~V}\right)$ and $\mathrm{B}\left(\mathrm{IF}_{1}\right.$ : $6.4 \mathrm{~V}$ ) separately. As the index of the degree of constancy the amount of difference between the matched brightness at 100 luxes illuminance level and other illuminance levels was adopted. The results of the analysis of variance are summarized in Tables la and $1 \mathrm{~b}$. It can be seen that the effect of $\mathrm{IF}_{2}$ upon the test field is not significant in the case of the darker $\mathrm{IF}_{\mathbf{1}}(1.6 \mathrm{~V})$, Condition $\mathrm{A}$, but significant at $5 \%$ level in the lighter $\mathbf{I F}_{1}$ $(6.4 \mathrm{~V})$, Condition B. On the other hand, the effect of illuminance is significant at $1 \%$ level in both conditions.

It is commonly claimed that if there are two or more articulated regions in the visual field they interact at the neuro-physiological level and inhibit each other. According to the recent experiments (Barlow, 1953; Hartline, 1956 and 1957) on the brightness response of the retina these interactions occur among the individual elements in the retina; e.g., among rods or cones, among bipolars, or among ganglion cells. 
What has been called brightness contrast or perceptual depression may be the result of lateral inhibition. According to this assumption the physiological events basic to the contrast are the followings; onfibers of the test field are inhibited by (a) the inducing on-fibers, (b) other on-fibers, of the test field, and (c) the spontancously discharging off-fibers (Diamond, 1960). Then, prior to the analysis of the present results in terms of the relationship between constancy and contrast, it seems necessary to compare the inhibitory effect of $\mathrm{IF}_{2}$ on the test field in Condition $\mathrm{A}$ with that in Condition B. According to the foregoing assumption, the following prediction seems possible; when the reflectance of $\mathrm{IF}_{1}$ is lower than the test field reflectance, there is little or no inhibitory effect of IF $_{1}$ on the test field, while its effect is strong when $I F_{1}$ has a higher reflectance than the test field; so the inhibitory effect of the outer inducing field, $\mathrm{IF}_{2}$, on the test field is greater in the former than in the latter, and consequently, change of the apparent brightness of the test field with the increase of $\mathrm{IF}_{2}$ reflectance is larger in Condition A than in Condition B. This implies for our results that the gradient of the curves should be greater under Condition $A$ than Condition B.

Now we must return to the relationship between constancy and contrast. As we have mentioned above, the effect of $\mathrm{IF}_{2}$ on constancy was significant at $5 \%$ level in Condition B, but was not significant in Condition A. If there is a co-variant relationship between constancy and contrast, the effect of $\mathrm{IF}_{2}$ should be in the same direction for both phenomena. The data obtained, however, contradict with this prediction. In fact, as contrast, the inhibitory effect of $\mathrm{IF}_{3}$ on the test field should be stronger in Condition A than B, but the results obtained showed the opposite. So we must conclude that the relationship between constancy and contrast is not necessarily covariant. These results of the present experiment agree with the findings of the previous study (Kozaki, 1963). Moreover, the present study suggests that for constancy such factor as the figure-ground relationship, which depends on the brightness gradient relationship between the test field and the inducing fields, is important.

\section{EXPERIMENT II}

\section{Purpose}

It has been found that judgments of brightness were strongly influenced by the structure of the visual field and the color appearance of the particular region (Katz, 1935; Katona, 1929; Hano, 1959; and Beck, 1964). Since, in our previous experiments, the stimuli were observed through the dark observation tube in the dark room, the structure of the visual field was fairly reduced, so that the test field tended to appear in the film color when it was surrounded by the darker inducing field. Our next concern is to find out whether or not earlier findings depend upon those conditions of the observation. The purpose of the present experiment is to examine this problem in conditions of everyday observation where the stimuli always appear as the surface color. Another purpose of the experiment is to test the effect of the test field area having the larger size than in the previous experiments (Kozaki, 1963, Exp. II).

\section{Apparatus}

The apparatus placed on the flat desk consisted of two parts; viewing box and light source box (Fig. 3). The large rectangular viewing box, from whose front side a rectangular window was cut, was covered with achromatic paper of $2.4 \%$ reflectance or $8.8 \%$. The light box containing four $300 \mathrm{w}$ reflector lamps and Hoya daylight filters was placed above the viewing box and out of $O$ 's line of sight. Illumination of the viewing box was provided through a diffusion glass, so that the illuminated surfaces appeared homogeneous. The intensity of illumination was changed by the controlled plates in which many small holes were opened. They were mounted between the 


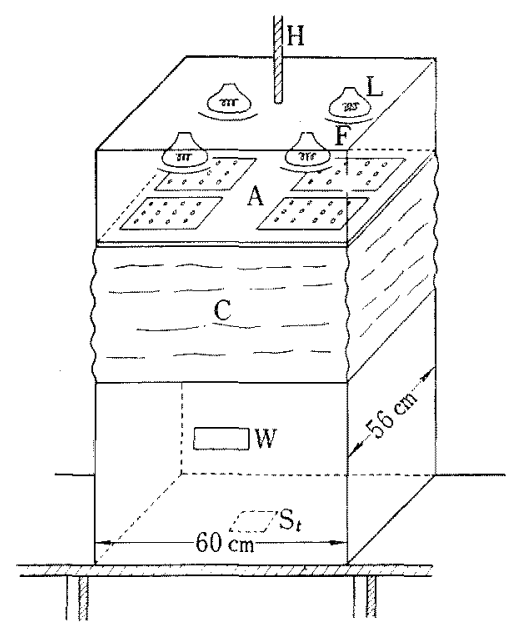

Fig. 3. Apparatus; St: Stimulus; W : Viewing window; C: Black cloth; L: Light source; F : Day-light filter; A : Adjustment plate of illuminance; $\mathbf{H}$ : Hanging cord.

lamps and the diffusion glass. A voltage regulator was used to keep the line voltage constant. The test field subtended a visual angle of $17^{\circ} 4^{\prime}$ or $5^{\circ} 48^{\prime}$, whereas the size of the background was kept at the constant visual angle of $52^{\circ} 12^{\prime} \times 60^{\circ} 12^{\prime}$. The test field had the lightness of the following levels: $1.6,2.2,3.6,5.0$, 6.4, 7.8 and 9.2 Munsell Value. The lightness of the background was black (2.4\% reflectance) or white $(88 \%$ reflectance). The intensity of illumination was kept at 500 or 100 luxes.

Each subject was seated before the chin rest in the dark room and told that through the aperture in front of the viewing box she would see a square stimulus in the center of the background and at the distance of $50 \mathrm{~cm}$ from her. Subject's task was to judge the brightness of the test field by the absolute judgments using 9 adjective categories from "very very dark, through 'medium' to 'very very light'. Two undergraduates of Tokyo Woman's Christian College served as $O$ s.

Results

Judgment categories used by $O_{\text {s }}$ were converted to the numerical scale ranging from 1 (very very dark) to 9 (very very light). Fig 4 presents the mean category difference between the brightness judg- ment of the test field at two different illuminance levels. In these plots, the law of brightness constancy would be represented by the line of zero slope at the zero on the ordinate axis. The results indicate that regardless of the size of the test field, the apparent brightness of the test field under the white background tends to exhibit greater constancy than the test field under the black background. Analyses of variance were separately performed for each size of the test field and for each subject, which showed that the effect of the background upon the apparent brightness of the test field was significant at $1 \%$ level. Fig. 4 and the results of analysis of variance reveal that as in the previous experiments the tendency toward constancy of the test field also depends upon the reflectance of the background under the stimulus field conditions such as the present experiment. That is, when the test field is surrounded by the lighter background than itself, the tendency toward constancy is higher, but when the background luminance is darker than that of the test field, the constancy is lower. The present results obtained under the same condition as everyday visual field coincide with the previous results which was obtained under the reduced stimulus conditions. So, the previous find-

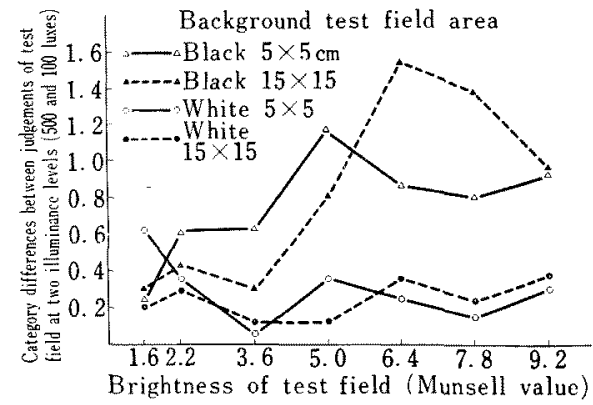

Fig. 4. The category differences between the judgments of the test field at the two illuminance level (500 and 100 luxes) as a function of the brightness of the test field are plotted with the background brightness and the area of the test field as the parameter. The mean of the data for $2 O \mathrm{~s}$ is plotted. 
ings about the effect of background reflectance upon constancy can be generalized beyond the reduced condition of the observation. In other words, the differences in the effect of the background reflectances on constancy are independent of the structure of the visual field.

Analysis of variance of the effect of the field area was separately done for each subject. It was found that for each subject and for each background the difference in the test field area did not have the significant effect. As would be expected on the basis of the previous findings (Kozaki, 1963, Exp. II), the factor of the test field area did not have definite effect upon constancy, even when the area of the test field was varied in the wider extent than in the previous experiment. Although the area of the test field may be the effective variable in contrast (Ogawa \& Kozaki, 1962; Torii \& Uemura, 1965), it is not effective in constancy.

\section{EXPERIMENT III}

\section{Purpose}

The findings of the previous study (1963) and Experiment I suggest that the explanation of constancy as brightness contrast which is the result of lateral inhibition in the retina is inadequate in predicting the tendency toward constancy of particular field, since brightness constancy is not a linear function of simultaneous contrast. The purpose of Experiment III is to investigate the effect of an additional stimulus, which was designed to exclude its inhibitory effect on the test field upon constancy.

\section{Apparatus and Procedure}

The apparatus and the main procedure were the same as in Experiment II. The stimuli consisted of the test field, the background, and the additional stimulus on the background which is at remote position from the test field. The walls of the viewing box were painted uniformly black. The square test field subtending a visual angle $4^{\circ} 38^{\prime}$ had the lightness of fifteen levels ranging from 1.6 to 8.5 Munsell Value. The reflectance of the background was kept constant at $19.77 \%$ (5 M.V., a neutral gray paper) throughout this experiment, and its visual angle was the same as in Experiment II. These stimuli were observed under three levels of illuminance, 30,600 , and 1200 luxes. The reflectance of the square additional stimulus subtending a visual angle $2^{\circ} 19^{\prime} \times 2^{\circ} 19^{\prime}$ which was set at the right or left upper position $(10 \mathrm{~cm}$ from the test field and $45^{\circ}$ against $O$ 's line of sight), was: $9.2 \mathrm{M.V}$. (Condition A), 1.6 M.V. (Condition B), and 9.2 and $1.6 \mathrm{M.V}$. (Condition C) in which the two stimuli having the different reflectance were simultaneously presented. Each experimental condition was compared to the control condition in which the test field was not accompanied by any additional stimuli. The method of the single stimuli was also adopted as in Experiment II. The procedure was identical with that of Experiment II, except that the $O$ was instructed to use the additional stimuli as a cue for brightness judgment of the test field. Data were obtained from six $O$ s who were undergraduate students of psychology at Tokyo Woman's Christian College.

Results

The results are shown in Fig. 5. In order to see the degree of the tendency toward constancy the mean category difference among the brightness judgments of the test field at three levels of illuminance was taken, and this was plotted against the brightness of the test field. In this figure the law of constancy is represented by the line of zero slope, a horizontal line, at the zero on the ordinate axis. The curves in Fig. 5 show the results under three additional stimulus conditions and control condition without an additional stimulus. The question which arises here is whether the additional stimulus has the effect on constancy of the apparent brightness of the test field; in particular, whether or not there is the difference in the effect on constancy under different reflectance conditions of the additional stimuli. In Fig. 5 it is observed that though the additional stimuli generally do not have a marked 


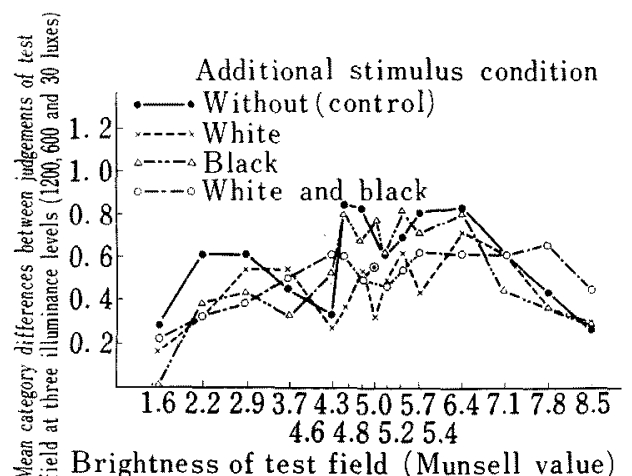

FIG. 5. The mean category differences among the judgments of the test field at three levels of illuminance $(1200,600$ and 30 luxes) are plotted against the brightness of the test field. The parameter on the curves are the conditions of the additional stimulus, Each datum is the mean of 6 Os.

effect on constancy, Condition A tends to exhibit greater constancy than the other conditions. This tendency is especially noticeable in comparing between curves under each experimental condition and control condition. The analysis of variance of the effect of the additional stimulus was summerized in Table 2, which shows that the effect of the additional stimulus on constancy is not significant in Conditions $\mathrm{B}$ and $\mathrm{C}$, but significant at $5 \%$ level in Condition $\mathrm{A}$ having the higher reflectance (white). The tendency toward constancy of the test field in Conditions B and $\mathrm{C}$ was similar to that of Control. It should be noted that addition of black stimulus (Con- dition B) and addition of both white and black stimuli at the same time (Condition C) did not have striking influence on brightness judgments of the field, but addition of white stimulus (Condition A) significantly affected in bringing about greater constancy than the Control Condition in which additional stimulus did not exist. An important aspect of Experiment III is that by the co-existence of higher reflectance stimulus, white, the tendency toward constancy of the test field is strengthened. Here, it should be recalled that the tendency toward constancy of the test field depends upon the reflectance of background, and above all, that the background of higher luminance than the test field plays an important role in producing the greater brightness constancy (Leibowitz, 1955; Kozaki, 1963). Findings in Experiment III fairly well coincide with these previous results. It seems that the coexisting stimulus of higher reflectance than that of the test field works as a cue tending to promote the tendency toward constancy, and whether the stimulus exists in the stimulus field as background or as additional stimulus does not matter much. It should be noted, however, that in spite of the existence of white stimulus the tendency toward constancy under Condition $\mathrm{C}$ was similar to that of Control and that there was not significant difference between both conditions. The reason for this is not clear, but it may be argued as follows. Condition $\mathrm{C}$ differs from Condition A only in that the test field was at the same time

TABLE 2

Analysis of variance for the effect of the additional stimulus on constancy in comparing between data under each experimental and control condition

\begin{tabular}{|c|c|c|c|c|c|c|c|c|c|c|c|c|}
\hline \multirow{2}{*}{ Source of variation } & \multicolumn{4}{|c|}{ Condition A } & \multicolumn{4}{|c|}{ Condition B } & \multicolumn{4}{|c|}{ Condition $\mathrm{G}$} \\
\hline & $S S$ & $d f$ & $M S$ & $F$ & $S S$ & $d f$ & $M S$ & $F$ & $S S$ & $d f$ & $M S$ & $F$ \\
\hline Additional field cond. (A) & 0.58 & 1 & 0.58 & $4.1^{*}$ & 0.029 & 1 & 0.029 & 0.576 & 0.132 & 1 & 0.132 & 0.95 \\
\hline Lightness of test field (L) & 4.03 & 14 & 0.288 & $2.05 *$ & 6.298 & 14 & 0.449 & $8.920 * *$ & 2.839 & 14 & 0.203 & 1.47 \\
\hline$A \times L$ & 0.71 & 14 & 0.051 & & 1.218 & 14 & 0.087 & 1.720 & 1.274 & 14 & 0.091 & \\
\hline $\mathrm{R}(\mathrm{AL})$ & 21.08 & 150 & 0.141 & & 7.545 & 150 & 0.050 & & 20.80 & 150 & 0.138 & \\
\hline
\end{tabular}


accompanied with two additional stimuli, white and black, as cues of brightness judgments. It therefore should be expected under Condition $\mathrm{C}$ that the brightness judgment of the test field was based on the interaction between white and black stimuli which occupy the end positions in gray scale (brightness scale). If that was the case, results in Condition $\mathrm{C}$ may not be altogether inconsistent with the greater constancy shown by white or higher reflectance stimulus.

\section{Discussion}

In the previous experiments (Kozaki, 1963) we examined systematically the relationship between constancy and contrast with respect to variables considered as the common parameters of both phenomena. The results indicated that there was not a linear relationship between brightness constancy and contrast, and that the change in contrast did not necessarily induce consistant change in constancy. The results of the present experiments provided more concrete evidence for the view that constancy does not vary as a linear function of contrast. In Experiment I the effects of the second inducing field on constancy of the test field did not appear to correspond to its inhibitory effects upon the test field. The results of Experiment II supported the previous finding that variation in the area of the test field did not change constancy while varying contrast. Moreover, the results of Experiment III, where additional stimulus was present but ineffective as inhibitor, revealed that the additional stimulus having high reflectance was effective for constancy. That is, irrespective of non-efficiency of its inhibitory effect, white additional stimulus tended to promote the tendency toward constancy. From all these experiments, we failed to support the assumption that there is a covariant relationship between constancy and contrast.

Leibowitz interpreted his experimental findings (1955, 1957 and 1961) as support- ing the view that simultaneous brightness contrast is one of the important mechanisms underlying the phenomenon of brightness constancy. Certainly his results demonstrated that both contrast and constancy covariantly increased with the increase of the luminance of the inducing field relative to that of the test field (1955), and with reduction of exposure duration (1957), and that both phenomena decreased with separation between test and inducing fields (1961). Our previous parametric study (Kozaki, 1963) showed, however, that variation in contrast (level of apparent brightness of the test field) could be obtained by varying inducing field reflectance without changing the slope of brightness as a function of illuminance (constancy), and that constancy was greatly promoted when the reflectance of the inducing field exceeded that of the test field. And, as recently pointed out by Freeman, the relationship between brightness contrast and exposure duration is so complex (Freeman, 1964) that care is needed in concluding the relationship between brightness constancy and contrast based on such variable as exposure duration. Furthermore, with respect to such variable as separation between test and inducing fields (Dunn \& Leibowitz 1961), it is questionable whether the decrease in constancy with increase of the spatial separation only depends upon the decrease in contrast. In the stimulus field condition used by Dunn \& Leiborvitz (1961), where a small test patch was set on the wall in the chamber uniformly painted flat black, the decrease in constancy with increase of separation between test and inducing fields may be based on some other alternation than contrast, e.g., the alternation of the color appearance of test patch (a film color or a surface color).

As we have shown above, there is little experimental confirmation of the covariant relationship between brightness constancy and contrast. In the experiments where supporting evidence was obtained, Helson, 1943; Leibowitz, 1955; 
Kozaki, 1963, and present experiments), it was also revealed that constancy was strongly influenced by the presence of white stimulus or higher reflectance stimulus other than the discriminative stimulus in the visual field. Why constancy is promoted by the co-existence of higher reflectance stimuli should be explored. For this problem, proportional law (Wallach, 1948) and adaptation-level theory (Helson, 1943, and 1964) have not offered sufficient explanation. And, as mentioned above, simultaneous contrast hypothesis was also unsuccessful in accounting for this problem. It seems that any explanation should note the fact that lighter stimuli play as important role in brightness constancy, perhaps by mediating the process of brightness judgments.

Though the assumption of co-variant relationship between brightness constancy and contrast did not hold, it does not necessarily imply that both phenomena are mutually unrelated. Probably, simulteneous contrast is one of the variables mediating brightness constancy (Beck, 1964). Yet whether or not simultaneous contrast is the basic mechanism subserving brightness constancy remains unanswered. Brightness constancy phenomenon appears to be a complex relational affairs involving the interaction of multiple situational factors, such as color appearance, relationship of luminance between the particular field and other fields (contrast), adaptation, and process of contour formation (more peripheral sensory process); stimulus configuration, set and memory (more central process), and factors relating to illumination judgments. How these factors cooperate in achieving approximate invariance of perceived brightness with variation in illumination is not yet clearly known. It is certainly possible that interaction of these multiple factors is a dynamic process, so that factors playing significant role mediating brightness constancy in some stimulus conditions may be unimportant in other conditions. It may be that multi- ple variables are weighted according to their efficiency for constancy in each stimulus situation. Exploring how these factors are mutually related in brightness constancy and clarifying the process of brightness judgment will be our concern in future.

\section{REFERENCES}

BARLOW, H. B. 1953 Summation and inhibition in the frog's retina. 7. Physiol., 119, 69-88.

ВЕск, J. 1964 The effect of gloss on perceived lightness. Amer. 7. Psychol., 27, 54-63.

Diamond, A. L. 1960 Theory of depression and enhancement in brightness response. Psychol. Rev., 67, 168-199.

Dunn, B. \& Leibowirz, H. 1961 The effect of separation between test and inducing fields on brightness constancy. F. exp. Psychol., 61, 505-507.

FREeman, R. B. Jr. 1964 The contrast interpretation of brightness constancy. Research Bull. No. 48, Pennsylvania State Univ.

Gelb, A, 1930 Die "Farbenkonstanz " der Sehdinge. In Bethe's Handbuch norm. path. Physiol,, 12, 1. Berlin: Springer Pp. 594-678.

Hano, A. 1959 The role of background in brightness constancy. Jap. F. Psychol. 30, 37-45 (In Japanese with English Abstract).

Hartline, H. K., Wagner, H. G. \& Ratliff F. 1956 Inhibition in the eye of Limulus. $\mathcal{F}$. gen. Physiol., 39, 651-673.

HARTLINe, H. K., \& RATLIFF, F. 1957 Inhibitory interaction of receptor units in the eye of the Limulus. F. gen. Physiol., 40, 357-376.

HeLson, H. 1938 Fundamental problems in color vision. I. The principle governing changes in hue, saturation, and lightness of nonselection samples in chromatic illumination. 7. exp. Psychol., 23, 430-476.

Helson, H. 1943 Some factors and implications of color constancy. 7. opt. Soc. Amer, 33, $555-567$.

Helson, H. 1964 Adaptation-Leve Theory. New York: Harper \& Row.

Kardos, L. 1934 Ding und Schatten. Z. Psychol., Erg-Bd.

Katona, G. 1929 Zur der Helligkeitskonstanz. Psychol. Forsch., 12, 94-126.

KATZ, D. 1935 The World of Color. London: Kegan Paul,

KorfKA, K. 1932 Some remarks on the theory of 
colour constancy. Psychol. Forsch., 16,329-354.

KOFFKA, K. 1932 A new theory of brightnessconstancy. A contribution to a general theory of vision. Report of a joint discussion on vision. $182-188$.

KoZAKI, A. 1963 A further study in the relationship between brightness constancy and contrast. Jap. psychol. Res., 5, 129-136.

LerBowrtz, H. 1955 The role of simultaneous contrast in brightness constancy. $\mathcal{J}$. exp. Psychol., 50, 15-19.

Leibowitz, H. \& Chinette, P. 1957 The effect of reduced exposure duration on brightness constancy. 7. exp. Psychol, 54, 49-53.

Ogawa, T. \& Kozaki, T. 1962 The effect of stimulus area on the apparent brightness. Proc. 26th Meeting of F.P.A, (In Japanese).

Torir, S. \& Uemura, Y. 1965 Effects of inducing luminance and area upon the apparent brightness of test field. Jap. psychol. Res., 7, 86-100.

WALLACH, H. 1948 Brightness constancy and the nature of achromatic colors. 7. exp. Psychol., 38, 310-324.

(Received Oct. 2, 1965) 